\title{
Quantum statistical treatment of Verlinde's conjecture in a Tsallis framework
}

\author{
A. Plastino ${ }^{1,3,4}$, M. C. Rocca ${ }^{1,2,3}$, \\ 1 Departamento de Física, Universidad Nacional de La Plata, \\ 2 Departamento de Matemática, Universidad Nacional de La Plata, \\ ${ }^{3}$ Consejo Nacional de Investigaciones Científicas y Tecnológicas \\ (IFLP-CCT-CONICET)-C. C. 727, 1900 La Plata - Argentina \\ ${ }^{4}$ SThAR - EPFL, Lausanne, Switzerland
}

December 20, 2018

\begin{abstract}
Verlinde has recently conjectured, via a Beckenstein-like thought experiment, that gravitation, instead of being an elementary force, is an emergent entropic one. This rather surprising conjecture was actually proved in [Physica A 505 (2018) 190], in a strictly classical statistical mechanics' environment. In this Communication, we work in a quantum statistical context to consider the conjecture in the case of bosons/fermions, in a Tsallis' framework. We prove that Tsallis' entropy is the operating potential energy in this quantum treatment, something that does not happen in the case of Boltzmann-Gibbs' entropy. In the classical limit, we show that the emergent force has a Newtonian dependence with the distance.
\end{abstract}

Keywords Gravitation, bosons, fermions, entropic force, emergent force, Verlide's conjecture. 


\section{Contents}

1 Introduction 3

1.1 Our goal ............................ 4

2 Entropic force for bosons in the microcanonical ensemble 4

2.1 Quantum entropic force .............. 4

2.2 Bose's entropic force in the classical limit $N / n \ll 1 \ldots 8$

3 Entropic force for fermions 9

3.1 Quantum entropic force for fermions . . . . . . . . . . . 9 9

3.2 Entropic force in the classical limit $N / n \ll 1 \ldots . . . . .11$

4 Conclusions

A Appendix 


\section{Introduction}

In 2011, Verlinde [1] conjectured a link between gravity and an entropic force. Such conjecture was proved correct recently citep1, in a phase-space, statistical mechanics' context.

Here we will confront two viewpoints

- Verlinde's elegant thought experiment, based in black-hole related assumptions, that leads to a Newtonian $r^{-2}$ radial dependence for the entropic force, with

- A quantum statistical mechanics treatment of a Fermi (Bose) gas that, in its classical limit, leads to the same radial dependence.

Verlinde suggests in his thought experiment that gravitation should emerge as a result of information about the positions of material particles, connecting a gravity's thermal treatment to 't Hooft's holographic principle. Accordingly, gravitation is to be regarded as an emergent phenomenon. The idea generated immense attention. See for instance [3, 4]. A very nice overview regarding the statistical mechanics of gravitation is to be encountered in Padmanabhan's work [5], and references therein.

This conjecture originated works in cosmology, the dark energy hypothesis, cosmological acceleration, cosmological inflation, and loop quantum gravity. The associated literature is extensive [4. A relevant input is due to Guseo [6], who demonstrated that the local entropy function, related to a logistic distribution, is a catenary and vice versa, an invariance interpreted through Verlindes conjecture regarding gravity as an entropic force. [6] puts forward a new interpretation of the local entropy in a system.

This paper does not deal with any of these issues, though. Considering that we proved Verlinde's conjecture in a classical context [2], we wish here to continue a discussion initiated in [7] with regards to the quantal bosons/fermions scenario. In [7] we used Boltzmann-Gibbs (BG) entropy. Here we wish to undertake a Tsallis-treatment. Why? Because distinctive advantages will be accrued in this way. It turns out that Tsallis entropy is a potential for the entropic force for $q=4 / 3$ (see proof in [2] and also in the Appendix), which is not the BG case. This makes Tsallis' entropy the natural information measure to link to gravitation. This should be natural enough, since it is well known that BG is the natural entropy for systems with short-range 
interaction, while Tsallis' is the one appropriate to long-range interactions [8].

We base our considerations on Chapters 6 and 7 of [9], to which the reader is referred for details. Only the microcanonical ensemble is used in this book, and thus here. It is assumed that each fermion or boson possesses an average energy $E / N$. Such average energy approximation produces results that, while approximate, describe important features of the ideal Fermi (Bose) gas [9]. In fact, most of the book is devoted to this excellent approximation, that allows one to appeal to the micro-canonical ensemble. This entails that the entropy is the logarithm of the multiplicity $\Omega$, according to the celebrated Boltzmann-formula.

\subsection{Our goal}

The present effort intends to contribute to the current debate/discussion regarding Verlinde's proposal, based on a thought-experiment, for an alternative (entropic) interpretation of gravity. A theory of quantum gravitation does not yet exist. What do we want to achieve here then? We can not expect to obtain en emerging entropic force that will yield classical gravitation in the quantum domain. What we wish to ascertain is whether the classical limit of our quantum statistical mechanics' Verlinde-treatment does yield Newton's gravitation in such limit. We will prove that such is the case. Thus, we contrast Verlinde's thought experiment with a rigorous statistical mechanics' argumentation. Such is the logic of the present effort.

\section{Entropic force for bosons in the microcanon- ical ensemble}

\subsection{Quantum entropic force}

We start by reminding the reader of the q-logarithm notion, defined according to [8] as

$$
\ln _{q} x=\frac{x^{1-q}-1}{1-q} .
$$

Tsallis entropy is defined. for a given set of micro-states labeled by $i$, whose probability is $P_{i}$, as 8 ] 


$$
S_{q}=-\sum_{i} P_{i}^{q} \ln _{q} P_{i}
$$

with $q$ any real number. An important portion of the immense Tsallis' literature [8] is devoted to ascertaining which is the appropriate value of $q$ in variegated scenarios. In our present environment we will see below that $q=4 / 3$.

We will use it to compute the multiplicity $\Omega$ for a Bose gas in the microcanonical ensemble following Boltzmann's logarithmic prescription in a Tsallisenvironment. Following [9], in a system of free bosons for which the number of accessible single-particle states is given by $n, \Omega$ can be thought of as the number of ways of distributing $N$ particles and $n-1$ "partitions" separating them [9]. It then reads [9]

$$
\Omega(E, V, N)=\frac{(N+n-1) !}{N !(n-1) !}=\frac{\Gamma(N+n)}{\Gamma(N+1) \Gamma(n)},
$$

where the energy $E$, the volume $V$, and the number of bosons $N$ are the extensive variables of the problem at hand. In terms of these variables one has [9]

$$
\frac{N}{n}=\frac{N}{V}\left(\frac{N}{E}\right)^{\frac{3}{2}}\left(\frac{3 h^{2}}{4 \pi e m}\right)^{\frac{3}{2}},
$$

an important relation that we will often employ below. $m$ stands for the gas' particles' mass. $e$ is Euler's number, and $h$ Planck's constant. The classical limit is attained for $N / n \ll 1$ [9]. Remember also that the $\Gamma$ function for large values of $z$ can be approximated by

$$
\Gamma(z) \approx \sqrt{2 \pi} z^{z-\frac{1}{2}} e^{-z}
$$

which we can use for $N>>1, n>>1$ to obtain

$$
\Omega(E, V, N) \approx \frac{(N+n)^{N+n}}{\sqrt{2 \pi} N^{N} n^{n}},
$$

so that the micro-canonical Tsallis' entropy becomes ( $k_{B}$ is Boltzmann's constant)

$$
\mathcal{S}_{q}=N k_{B} \ln _{q} \Omega(E, V, N)^{\frac{1}{N}}
$$


For $q \rightarrow 1$ one has

$$
\mathcal{S}=N k_{B} \ln \Omega(E, V, N)^{\frac{1}{N}}=k_{B} \ln \Omega(E, V, N) .
$$

Thus, for $q \rightarrow 1$ Tsallis's microcanonical entropy in terms of the multiplicity becomes Boltzmann's celebrated one.

Now, it was seen in [2] that the gravitational interaction can be extracted, out of the infinite family of different Tsallis' entropies associated to all possible $q$-values, only for $q=\frac{4}{3}$. For other $q$-values the gradient of $S_{q}$ becomes proportional to $1 / r^{\nu}$ with $\nu \neq 2$ [2]. See Appendix for details. Thus, we are heuristically forced to select $q=4 / 3$. Note that, for each value of $q$, Tsallis has introduced a different statistical mechanics. For example, for $q=1$ we obtain the orthodox statistical mechanics of Boltzmann-Gibbs. What Tsallis did it is not to define just a new (single) realization of statistical mechanics, but a new infinite set of different statistical mechanics' realizations [8]. Accordingly,

$$
\mathcal{S}_{\frac{4}{3}}=3 N k_{B}\left(1-\Omega^{-\frac{1}{3 N}}\right),
$$

and using again (2.4) we write

$$
n=V\left(\frac{E}{N}\right)^{\frac{3}{2}}\left(\frac{4 \pi e m}{3 h^{2}}\right)^{\frac{3}{2}} .
$$

It is important to realize that here we find that

$$
n \propto V .
$$

It is seen possible at this point to cast $\Omega$ in the fashion

$$
\Omega=\frac{e^{\gamma}}{\sqrt{2 \pi} N^{N}},
$$

with

$$
\begin{gathered}
\gamma=\left[N+V\left(\frac{E}{N}\right)^{\frac{3}{2}}\left(\frac{4 \pi e m}{3 h^{2}}\right)^{\frac{3}{2}}\right] \ln \left[N+V\left(\frac{E}{N}\right)^{\frac{3}{2}}\left(\frac{4 \pi e m}{3 h^{2}}\right)^{\frac{3}{2}}\right]- \\
{\left[V\left(\frac{E}{N}\right)^{\frac{3}{2}}\left(\frac{4 \pi e m}{3 h^{2}}\right)^{\frac{3}{2}}\right] \ln \left[V\left(\frac{E}{N}\right)^{\frac{3}{2}}\left(\frac{4 \pi e m}{3 h^{2}}\right)^{\frac{3}{2}}\right]}
\end{gathered}
$$

or 


$$
\gamma=(N+n) \ln (N+n)-n \ln n
$$

The entropy's gradient becomes then

$$
\vec{\nabla} \mathcal{S}_{\frac{4}{3}}=k_{B} \Omega^{-\frac{1}{3 N}} \vec{\nabla} \gamma
$$

Now, since $V=\frac{4}{3} \pi r^{3}$ this entails

$$
\frac{\partial \mathcal{S}_{\frac{4}{3}}}{\partial r}=k_{B} \Omega^{-\frac{1}{3 N}} \frac{\partial \gamma}{\partial r},
$$

since $\mathcal{S}_{\frac{4}{3}}$ depends just upon $r$. Thus,

$$
\frac{\partial \mathcal{S}_{\frac{4}{3}}}{\partial r}=k_{B} 4 \pi r^{2} \Omega^{-\frac{1}{3 N}} \frac{\partial \gamma}{\partial V} .
$$

Taking into account that

$$
\begin{gathered}
\frac{\partial \gamma}{\partial V}=\left(\frac{E}{N}\right)^{\frac{3}{2}}\left(\frac{4 \pi e m}{3 h^{2}}\right)^{\frac{3}{2}}\left\{\ln \left[N+V\left(\frac{E}{N}\right)^{\frac{3}{2}}\left(\frac{4 \pi e m}{3 h^{2}}\right)^{\frac{3}{2}}\right]-\right. \\
\left.\ln \left[V\left(\frac{E}{N}\right)^{\frac{3}{2}}\left(\frac{4 \pi e m}{3 h^{2}}\right)^{\frac{3}{2}}\right]\right\},
\end{gathered}
$$

and remembering Verlinde's definition for the entropic force [1] we find

$$
\vec{F}_{e}=-\lambda k_{B} T \vec{\nabla} \mathcal{S}_{\frac{4}{3}}
$$

or the nice result

$$
F_{e}=-\lambda k_{B} T \frac{\partial \mathcal{S}_{\frac{4}{3}}}{\partial r},
$$

that by appeal to (2.18) yields

$$
\begin{gathered}
F_{e}=-\frac{\lambda}{\beta} 4 \pi r^{2} \Omega^{-\frac{1}{3 N}}\left(\frac{E}{N}\right)^{\frac{3}{2}}\left(\frac{4 \pi e m}{3 h^{2}}\right)^{\frac{3}{2}}\left\{\ln \left[N+V\left(\frac{E}{N}\right)^{\frac{3}{2}}\left(\frac{4 \pi e m}{3 h^{2}}\right)^{\frac{3}{2}}\right]-\right. \\
\left.\ln \left[V\left(\frac{E}{N}\right)^{\frac{3}{2}}\left(\frac{4 \pi e m}{3 h^{2}}\right)^{\frac{3}{2}}\right]\right\}
\end{gathered}
$$


or

$$
\begin{gathered}
F_{e}=-\frac{\lambda}{\beta} 4 \pi r^{2} \Omega^{-\frac{1}{3 N}}\left(\frac{E}{N}\right)^{\frac{3}{2}}\left(\frac{4 \pi e m}{3 h^{2}}\right)^{\frac{3}{2}}\left\{\ln \left[N+\frac{4 \pi r^{3}}{3}\left(\frac{E}{N}\right)^{\frac{3}{2}}\left(\frac{4 \pi e m}{3 h^{2}}\right)^{\frac{3}{2}}\right]-\right. \\
\left.\ln \left[\frac{4 \pi r^{3}}{3}\left(\frac{E}{N}\right)^{\frac{3}{2}}\left(\frac{4 \pi e m}{3 h^{2}}\right)^{\frac{3}{2}}\right]\right\},
\end{gathered}
$$

that can also be cast as

$$
F_{e}=-\frac{\lambda}{\beta} 4 \pi r^{2} \Omega^{-\frac{1}{3 N}}\left(\frac{E}{N}\right)^{\frac{3}{2}}\left(\frac{4 \pi e m}{3 h^{2}}\right)^{\frac{3}{2}} \ln \left[1+\frac{N}{V}\left(\frac{N}{E}\right)^{\frac{3}{2}}\left(\frac{3 h^{2}}{4 \pi e m}\right)^{\frac{3}{2}}\right] .
$$

Note that $F_{e}$ does not diverge at the origin but vanishes there. However, this happens at distances to the origin of the order of one hundredth of the Planck-length. No practical consequences can be detected, though. Minding (2.4) we also have

$$
F_{e}=-\frac{\lambda}{\beta} 4 \pi r^{2} \Omega^{-\frac{1}{3 N}}\left(\frac{E}{N}\right)^{\frac{3}{2}}\left(\frac{4 \pi e m}{3 h^{2}}\right)^{\frac{3}{2}} \ln [1+N / n] .
$$

Notice also that the entropic force vanishes at zero temperature and diverges when $T \rightarrow \infty$. This putatively happened at the Big-Bang. There we have $r=0$ as well, so that the behavior of $F_{e}$ is complicated. However, this does not matter because at these limits quantum gravity, unknown today, reigns.

A word of caution is necessary here. Since a theory of quantum gravity does not exist yet, we must not naively think that these equations for $F_{e}$ can be taken at face value. What is really of interest here is just the classical limit of $F_{e}$, that we are going to discuss below.

\subsection{Bose's entropic force in the classical limit $N / n \ll 1$}

The idea is to judiciously employ (2.23) and (2.24) in this limit. From (2.4), i.e., $\frac{N}{n}=\frac{N}{V}\left(\frac{N}{E}\right)^{\frac{3}{2}}\left(\frac{3 h^{2}}{4 \pi e m}\right)^{\frac{3}{2}}$, plus $V=4 \pi r^{3} / 3$, one sees that

$$
N / n \ll 1 \rightarrow r>>1 .
$$

From (2.4) we also ascertain that we can replace the logarithm by its argument minus unity in (2.24), that then becomes 


$$
F_{e}=-\frac{\lambda}{\beta} 4 \pi r^{2} \Omega^{-\frac{1}{3 N}}\left(\frac{E}{N}\right)^{\frac{3}{2}}\left(\frac{4 \pi e m}{3 h^{2}}\right)^{\frac{3}{2}}\left[\frac{N}{V}\left(\frac{N}{E}\right)^{\frac{3}{2}}\left(\frac{3 h^{2}}{4 \pi e m}\right)^{\frac{3}{2}}\right]
$$

and

$$
F_{e}=-\frac{\lambda}{\beta} 4 \pi r^{2} \Omega^{-\frac{1}{3 N}} \frac{N}{V} .
$$

Now, according to (2.11) we have $n \propto V$ and (2.6) entails that in our limit we have

$$
\Omega \propto V^{N} .
$$

. Thus,

$$
\Omega^{-\frac{1}{3 N}} \propto\left(1 / V^{1 / 3}\right),
$$

. so that $F_{e}$ becomes

$$
F_{e} \propto-r^{2},
$$

where the proportionality constant is assumed to include Newton's gravitation constant $G$. This proves (in statistical mechanics' fashion), for free bosons, Verlinde's second conjecture: in the classical limit, the corresponding entropic force decreases as $1 / r^{2}$, like Newton's gravitation [2]. This dependence of $F_{e}$ with $r$ is all what Verlinde actually proved in [1, in a Beckensteinlike thought-experiment. There he assumes the number of bits $N$ contained in an appropriate Beckenstein enfolding screen can be cast as $N=A c^{3} / G \hbar$,

with $A$ the screen's area. Actually, this is, a priori, Verlinde's definition of $G$, that later will turn out to be gravitation's constant. Summing up, neither in Verlinde's derivation nor in ours we see $G$ emerging from first principles. It is introduced in an ad hoc fashion, "by hand".

\section{$3 \quad$ Entropic force for fermions}

\subsection{Quantum entropic force for fermions}

Here we deal with $N$ fermions and $n$ micro-states that can be occupied by just one fermion. We have a multiplicity $\Omega$ given by $[9]$

$$
\Omega=\frac{n !}{(n-N) ! N !}=\frac{\Gamma(n+1)}{\Gamma(n-N+1) \Gamma(N+1)} .
$$


For $N>>1$ and $n>>1$ one is allowed to write

$$
\Omega=\frac{e}{\sqrt{2 \pi}} \frac{(n+1)^{n+1}}{(n-N+1)^{n-N+1}(N+1)^{N+1}},
$$

that can be recast as

$$
\Omega=\frac{e}{\sqrt{2 \pi}} e^{\gamma}
$$

where $\gamma$ is

$$
\gamma=(n+1) \ln (n+1)+(N-n-1) \ln (n+1-N)-(N+1) \ln (N+1) .
$$

The derivative of $\gamma$ with respect to $V$ is

$$
\begin{gathered}
\frac{\partial \gamma}{\partial V}=\left(\frac{E}{N}\right)^{\frac{3}{2}}\left(\frac{4 \pi e m}{3 h^{2}}\right)^{\frac{3}{2}}\left\{\ln \left[1+V\left(\frac{E}{N}\right)^{\frac{3}{2}}\left(\frac{4 \pi e m}{3 h^{2}}\right)^{\frac{3}{2}}\right]-\right. \\
\left.\ln \left[1+V\left(\frac{E}{N}\right)^{\frac{3}{2}}\left(\frac{4 \pi e m}{3 h^{2}}\right)^{\frac{3}{2}}-N\right]\right\} .
\end{gathered}
$$

Retracing now here the boson-steps of the preceding Section we find

$$
\begin{gathered}
F_{e}=-\frac{\lambda}{\beta} 4 \pi r^{2} \Omega^{-\frac{1}{3 N}}\left(\frac{E}{N}\right)^{\frac{3}{2}}\left(\frac{4 \pi e m}{3 h^{2}}\right)^{\frac{3}{2}}\left\{\ln \left[1+V\left(\frac{E}{N}\right)^{\frac{3}{2}}\left(\frac{4 \pi e m}{3 h^{2}}\right)^{\frac{3}{2}}\right]-\right. \\
\left.\ln \left[1+V\left(\frac{E}{N}\right)^{\frac{3}{2}}\left(\frac{4 \pi e m}{3 h^{2}}\right)^{\frac{3}{2}}-N\right]\right\} .
\end{gathered}
$$

Comparing (2.23) for bosons with (3.6) for fermions we see that they are not identical This does not matter, though, since a theory of quantum gravity is not available yet and we should not take the above cited equations at face value. What matters are their classical limits and they do coincide, of course.Finally, we can also cast (3.6) as

$$
F_{e}=-\frac{\lambda}{\beta} 4 \pi r^{2} \Omega^{-\frac{1}{3 N}}\left(\frac{E}{N}\right)^{\frac{3}{2}}\left(\frac{4 \pi e m}{3 h^{2}}\right)^{\frac{3}{2}}\left\{\ln \left[1+\frac{4 \pi r^{3}}{3}\left(\frac{E}{N}\right)^{\frac{3}{2}}\left(\frac{4 \pi e m}{3 h^{2}}\right)^{\frac{3}{2}}\right]-\right.
$$




$$
\left.\ln \left[1+\frac{4 \pi r^{3}}{3}\left(\frac{E}{N}\right)^{\frac{3}{2}}\left(\frac{4 \pi e m}{3 h^{2}}\right)^{\frac{3}{2}}-N\right]\right\} .
$$

Notice that one has, according to the last equation,

$$
1+\frac{4 \pi r^{3}}{3}\left(\frac{E}{N}\right)^{\frac{3}{2}}\left(\frac{4 \pi e m}{3 h^{2}}\right)^{\frac{3}{2}}-N>0,
$$

so that there is a lower bound for $r$ since Eq. (3.8) entails

$$
r>\left[\frac{3(N-1)}{4 \pi}\right]^{\frac{1}{3}}\left(\frac{N}{E}\right)^{\frac{1}{2}}\left(\frac{3 h^{2}}{4 \pi e m}\right)^{\frac{1}{2}} .
$$

Selecting $m=$ uranium's mass, $N=500, v=0.1 c$ ( $c=$ speed of light) we obtain: $r=2 \cdot 410^{-21} \mathrm{~m}$. This might perhaps suggest a kind of space-quantization?. Notice also that the entropic force vanishes at zero temperature. if we select $\lambda$ independent of $T$.

\subsection{Entropic force in the classical limit $N / n \ll 1$}

First of all we realize that (2.25) holds in this situation too. We approximate things now for the classical limit, starting with (3.7), in the fashion

$$
\begin{gathered}
F_{e}=-\frac{\lambda}{\beta} 4 \pi r^{2} \Omega^{-\frac{1}{3 N}}\left(\frac{E}{N}\right)^{\frac{3}{2}}\left(\frac{4 \pi e m}{3 h^{2}}\right)^{\frac{3}{2}}\left\{\ln \left[V\left(\frac{E}{N}\right)^{\frac{3}{2}}\left(\frac{4 \pi e m}{3 h^{2}}\right)^{\frac{3}{2}}\right]-\right. \\
\left.\ln \left[V\left(\frac{E}{N}\right)^{\frac{3}{2}}\left(\frac{4 \pi e m}{3 h^{2}}\right)^{\frac{3}{2}}-N\right]\right\}
\end{gathered}
$$

or

$$
F_{e}=\frac{\lambda}{\beta} 4 \pi r^{2} \Omega^{-\frac{1}{3 N}}\left(\frac{E}{N}\right)^{\frac{3}{2}}\left(\frac{4 \pi e m}{3 h^{2}}\right)^{\frac{3}{2}} \ln \left[1-\frac{N}{V}\left(\frac{N}{E}\right)^{\frac{3}{2}}\left(\frac{3 h^{2}}{4 \pi e m}\right)^{\frac{3}{2}}\right]
$$

that also reads

$$
F_{e}=\frac{\lambda}{\beta} 4 \pi r^{2} \Omega^{-\frac{1}{3 N}}\left(\frac{E}{N}\right)^{\frac{3}{2}}\left(\frac{4 \pi e m}{3 h^{2}}\right)^{\frac{3}{2}} \ln [1-N / n] .
$$


Expanding now the logarithm we arrive at

$$
F_{e}=\frac{\lambda}{\beta} 4 \pi r^{2} \Omega^{-\frac{1}{3 N}}\left(\frac{E}{N}\right)^{\frac{3}{2}}\left(\frac{4 \pi e m}{3 h^{2}}\right)^{\frac{3}{2}}\left[-\frac{N}{V}\left(\frac{N}{E}\right)^{\frac{3}{2}}\left(\frac{3 h^{2}}{4 \pi e m}\right)^{\frac{3}{2}}\right]
$$

or, for the entropic force in the classical limit (CL)

$$
F_{e}(C L)=-(N / V) \frac{\lambda}{\beta} 4 \pi r^{2} \Omega^{-\frac{1}{3 N}}
$$

It is of the essence now to ascertain the behavior of $\Omega$ in the classical limit. Thus, we focus attention upon $\gamma$. For this, we go back to (2.14) at this point and realize, that in the classical limit, it reduces to

$$
\gamma \approx N \ln (n)
$$

Thus, according to (3.3),

$$
\Omega \propto \exp \gamma=n^{N}
$$

Further, we have

$$
n \propto V
$$

, so that

$$
\Omega \propto V^{N}
$$

Accordingly

$$
\Omega^{-\frac{1}{3 N}} \propto V^{-\frac{1}{3}},
$$

which finally yields, for the entropic force in the classical limit

$$
F_{e} \propto-\frac{1}{r^{2}}
$$

We have encountered a similar expression for the emergent entropic force in the classical limit similar to that obtained for bosons. 


\section{Conclusions}

Verlinde conjectured in 2011 that gravitation, instead of being an elementary force, is an emergent entropic one. This rather surprising conjecture had 3280 downloads and 717 cites in ArXiv! In a phase-space classical context it was actually proved true in [2].

Here we asked for its workings in a quantum scenario and it was proved again, this time in the classical limit of the quantum treatment. The relevant question is now: what kind of entropy yields gravitation as an entropic force?

We responded in this work that such an entropy is Tsallis' one for $q=4 / 3$ (an heuristically choice of $q$ ), both for fermions and for bosons.

Remark that, for fermions, we have found a lower bound for the distance to the origin $r$. 


\section{References}

[1] E. Verlinde, arXiv1001.0785 [hep-th]; JHEP 04 (2011) 29.

[2] A. Plastino, M. C. Rocca Physica A 505 (2018) 190.

[3] D. Overbye, A Scientist Takes On Gravity, The New York Times, 12 July 2010; M. Calmthout, New Scientist 205 (2010) 6.

[4] J. Makela, arXiv1001.3808v3; J. Lee, arXiv1005.1347; V. V. Kiselev, S. A. Timofeev , Mod. Phys. Lett. A 25 (2010) 2223; T. Aaltonen et al; Mod. Phys. Lett. A 25 (2010) 2825.

[5] T. Padmanabhan, arXiv 0812.2610v2.

[6] R. Guseo, Physica A 464 (2016) 1.

[7] A. Plastino, M. C. Rocca, G. L. Ferri: Physica A 511 (2018) 139

A. Plastino, M. C. Rocca: https://www.researchgate.net/publication/324831972_Spati

[8] M. Gell-Mann and C. Tsallis, Eds. Nonextensive Entropy Interdisciplinary applications, Oxford University Press, Oxford, 2004; C. Tsallis, Introduction to Nonextensive Statistical Mechanics Approaching a Complex World, Springer, New York, 2009; See also http//tsallis.cat.cbpf.br/biblio.htm for a regularly updated bibliography on the subject.

[9] D. S. Lemons, A Student's Guide to Entropy, Cambridge University Press (2014). 


\section{A Appendix}

\section{Tsallis' q-entropy of the free particle}

Tsallis' q-partition function for a free particle of mass $m$ in $\nu$ dimensions reads 8

$$
\mathcal{Z}_{\nu}=V_{\nu} \int\left[1+(1-q) \beta \frac{p^{2}}{2 m}\right]_{+}^{\frac{1}{q-1}} d^{\nu} p,
$$

with the particle probability distribution $\xi(p)$ being

$$
\xi=\frac{1}{\mathcal{Z}_{\nu}}\left[1+(1-q) \beta \frac{p^{2}}{2 m}\right]_{+}^{\frac{1}{q-1}},
$$

where $V_{\nu}$ is the volume of an hypersphere in $\nu$ dimensions and we assume $q>1$. A.1 can be recast as

$$
\mathcal{Z}_{\nu}=\frac{2 \pi^{\frac{\nu}{2}}}{\Gamma\left(\frac{\nu}{2}\right)} V_{\nu} \int_{0}^{\infty}\left[1+(1-q) \beta \frac{p^{2}}{2 m}\right]_{+}^{\frac{1}{q-1}} p^{\nu-1} d p .
$$

With the change of variables $x^{2}=\frac{p^{2}}{2 m}$ one has

$$
\mathcal{Z}_{\nu}=\frac{(2 m \pi)^{\frac{\nu}{2}}}{\Gamma\left(\frac{\nu}{2}\right)} V_{\nu} \int_{0}^{\frac{1}{(q-1) \beta}}[1+(1-q) \beta x]^{\frac{1}{q-1}} x^{\frac{\nu}{2}-1} d x
$$

that after integration becomes

$$
\mathcal{Z}_{\nu}=V_{\nu}\left[\frac{(2 m \pi)}{(q-1) \beta}\right]^{\frac{\nu}{2}} \frac{\Gamma\left(\frac{q}{q-1}\right)}{\Gamma\left(\frac{q}{q-1}+\frac{\nu}{2}\right)} .
$$

The mean energy is

$$
<\mathcal{U}_{\nu}>=\frac{V_{\nu}}{\mathcal{Z}_{\nu}} \int\left[1+(1-q) \beta \frac{p^{2}}{2 m}\right]_{+}^{\frac{1}{q-1}} \frac{p^{2}}{2 m} d^{\nu} p
$$

or

$$
<\mathcal{U}_{\nu}>=\frac{V_{\nu}}{\mathcal{Z}_{\nu}} \frac{(2 m \pi)^{\frac{\nu}{2}}}{\Gamma\left(\frac{\nu}{2}\right)} \int_{0}^{\frac{1}{(q-1) \beta}}[1+(1-q) \beta x]^{\frac{1}{q-1}} x^{\frac{\nu}{2}} d x
$$


so that after integration we find

$$
<\mathcal{U}>_{\nu}=\frac{\nu}{2(q-1) \beta} \frac{\Gamma\left(\frac{1}{q-1}+\frac{\nu}{2}+1\right)}{\Gamma\left(\frac{1}{q-1}+\frac{\nu}{2}+2\right)},
$$

and finally

$$
<\mathcal{U}>_{\nu}=\frac{\nu}{[2 q+\nu(q-1)] \beta} .
$$

For the entropy one has [8]

$$
\mathcal{S}_{\nu}=\ln _{q} \mathcal{Z}_{\nu}+\mathcal{Z}_{\nu}^{1-q} \beta<\mathcal{U}>_{\nu}
$$

\section{The Tsallis entropic force}

We specialize things now to $\nu=3$ and $q=\frac{4}{3}$. Why do we select this special value $q=\frac{4}{3}$ ? There is a solid reason. This is because

$$
\mathcal{S}_{\nu}=\ln _{q} \mathcal{Z}_{\nu}+\mathcal{Z}_{\nu}^{1-q} \beta<\mathcal{U}>_{\nu}
$$

Since the entropic force is to be defined as proportional to the gradient of $\mathcal{S}$, there is a unique $q$-value for which the dependence on $r$ of the entropic force is $\sim r^{-2}$ when $\nu=3$. Thus we obtain, for $q=4 / 3$,

$$
\begin{gathered}
\mathcal{Z}=\left(\frac{6 m \pi}{\beta}\right)^{\frac{3}{2}} \frac{8 \pi}{\Gamma\left(\frac{11}{2}\right)} r^{3}, \\
<\mathcal{U}>=\frac{9}{11 \beta} .
\end{gathered}
$$

Following Verlinde [1] we define the entropic force as

$$
\overrightarrow{\mathcal{F}}_{e}=-\frac{\lambda(m, M)}{\beta} \vec{\nabla} \mathcal{S},
$$

where $\lambda$ is a numerical parameter depending on the masses involved, $m$ and a new one $M$ that we place at the center of the sphere. Thus,

$$
\overrightarrow{\mathcal{F}}_{e}=-\frac{24}{11}\left[\frac{\Gamma\left(\frac{11}{1}\right)}{8 \pi}\right]^{\frac{1}{3}}\left(\frac{k_{B} T}{6 m \pi}\right)^{\frac{1}{2}} \frac{\lambda(m, M)}{r^{2}} \vec{e}_{r}
$$


where $\vec{e}_{r}$ is the radial unit vector. We see that $F_{e}$ acquires an appearance quite similar to that of Newton's gravitation, as conjectured by Verlinde en [1. Note that entropic force vanishes at zero temperature, in agreement with Thermodynamics' third law. 